\title{
Motion Detector Security System for Indoor Geolocation
}

\author{
Nosiri O.C., Akwiwu-Uzoma C.C., Nmaju U.A., Elumeziem C.H.
}

\begin{abstract}
This paper focused on the development of a microcontroller based security system for indoor geo-location using motion detector. The system carried out intruder detection that reinforces surveillance technology to provide essential security with associated control and alert operations. The security pivots on the integration of cameras and motion detectors into web application. The Raspberry $\mathrm{Pi}$, a smart surveillance system, takes input from the motion detector and controls (actuates) the pi camera for remote sensing and surveillance, sends the video to a web server which allows the user or homeowner to access the videos using a web application. The system, on intrusion alerts the owner by SMS, buzzes the alarm located at a convenient distant. The designed security system is characterized with efficient video camera for remote sensing and surveillance, featured with stream live video and records for subsequent replay and offers a cost effective ubiquitous surveillance solution, efficient and easy to implement.
\end{abstract}

\section{Index Terms - Raspberry Pi, PIR Sensor, Motion detector}

\section{INTRODUCTION}

In the world day, researchers and developers have come up with a wide range of surveillance systems that are deployed at homes, in industries and remote areas at the same time control the tasks through affordable, intelligent and easy-to-implement hardware and software systems. Some have so far been realized while others still remain a work in progress.

Criminal activities is becoming a very dexterous act nowadays due to intelligent and novel methods criminals manoeuvre to succeed in their activities in residential apartments. One of the most important things a person desires is the safety of the family and home. From global perspectives, the rising trend of criminal activities is becoming very alarming and has created much public outcry to government for urgent mitigation measures. This uproar has been characterized of diverse chaos ranging from human abduction, robbery attack, political bedlam, terrorism, herdsmen invasion and etcetera.

A security system is a combination of hardware and software that protect life and properties in both residential and commercial buildings from intrusion. The history of security system is interesting because the desire for this protection and security dates back thousands of years ago. In [1] stated that the idea of security systems came about after it was perceived that value of life and property could be lost suddenly either through fire, robbery, force etc., without a prior warning/information. Over decades, security systems have changed from simple control panels and locks into high-technological gadgets.

Nosiri Onyebuchi Chikezie, Akwiwu-Uzoma Chukwuebuka, Nmaju Uche Anyauba, Elumeziem Chika Hope, Department of Electrical and Electronic Engineering, Federal University of Technology, Owerri, Nigeria.
In today's home security systems, the trend of progress is very overwhelming due to the habitual novel approaches the mobsters deploy to penetrate homes. One of the challenging facts about some security systems like home surveillance cameras is that it has the capacity to only record the incident and could not be able to carry out other intelligent online and data processing functions like its ability to detect motions with instant frame configuration of the motions, store the captured video through a web server with additional short message services to the intended recipient.

It is perceived that not all families could afford the high cost of installation of the security surveillance system in the market today, this study considered designing a cost effective and dynamic motion detector security system based on Raspberry Pi microcontroller. The system will be able to detect motion (intruder), activate the embedded camera to take frames of video after motion is sensed and store the video on a web server with SMS alert to the facility owner. The hybridized video surveillance system with motion sensing technology is believed to revolutionize the perception of security systems on the cost of power reduction for the camera and with increased efficiency.

\section{II.LITERATURE REVIEW}

A GSM Based Home Security Alarm System Using Arduino was implemented by [2]. The proposed system consists of a PIR Motion sensor, GSM module and the Arduino microcontroller. The system works as follows: as the PIR sensor detects any motion, the output of the sensor goes HIGH. This is detected by the Arduino, it then communicates with the GSM module via serial communication to make a call to the pre-programmed mobile number. The project was primarily designed for detecting intruders and informing the owner by phone calls. The advantage of this system is that it is simple and affordable. But it lacks efficiency because if there is traffic congestion in the mobile network, there could be possibility of poor call initialization and the owner could not be signaled on the intrusion.

The authors of [3] designed and implemented a low cost smart security camera with night vision capability using Raspberry Pi with Passive infrared motion sensor. The Raspberry pi and passive infrared sensor (PIR sensor) handles the moving body, control algorithms for the alarms and sends captured pictures to user's e-mail. As part of its alarm system the speech sounds "intruder" is played when there is detection through a loud speaker. The system uses ordinary web camera but its infra-red filter was removed in order to have night vision capability. With help of light dependent resistor, it will sense whether it is night or day if it is night the led will ON when it detect intruder. The advantage of the system is that it takes into account day and night periods using the light dependent resistor to keep the system active at all times, it also included a night vision capability. 
The "Global System for Mobile Communication (GSM) Based Home Security System with SMS Alert Using Human Body Motion Detective and GSM Module", developed by [4], was a system implemented which comprised of an infrared motion detector and magnetic sensor as a transducer for detecting intruder's motion or break-in through a door. The signals were processed by an embedded microcontroller unit which activated the GSM module and send SMS message to the house owner's mobile phone, while triggering an alarm in the location. The advantages the system had was in cost effectiveness and remote alertness but lacks the streaming video coverage which is essential to clearly identify the intruder(s) and possibly apprehend them.

The "Home Alarm System using Detector Sensor" was proposed by [5]. The work deployed Passive Infrared (PIR) sensors, buzzer, a timer circuit (555 timer) and recording unit in the design. The authors efficiently employed the use of multiple sensors for efficient detection with Buzzers remotely installed. Its video is only stored locally, not internet enabled. This remains one of the limitations of the system because the location of the device where the video is stored and recorded could be identified and destroyed.

Several criteria have been used to select a security system required to safeguard a facility. The chief among all these has been the cost of implementation, remote monitoring and efficiency. The Raspberry $\mathrm{Pi}$ as a microcomputer has immense functionality. The choice of the Raspberry pi gives room for fast, reliable, cost-effective and remote surveillance system. One distinguished feature of this research work over all other pi dependent surveillance systems is the 'triple play' access to the video or picture feed of the pi. Two of those being internet based and remote access from anywhere in the world, and the other being the actual storage of the feed to the pi local storage. The system, on intrusion alerts the owner by SMS, buzzes the alarm located at a convenient distant and record feeds which could be accessed in 3 distinct ways. The system is characterized with efficient video camera for remote sensing and surveillance, featured with stream live video and records for subsequent replay.

This section introduces a brief description of the system design major components which include(a) the Raspberry Pi 3 Model B +, (b) PIR motion sensors, (c) Pi camera module, (d) Micro SD card (e) Buzzer and (f) Web server

\section{(a) Raspberry Pi 3 Model B+}

For the microcontroller (Raspberry pi) based security system using motion detector to be utilized for effective monitoring and alerting, the system has to provide at least three functions. These functions are: detection, video recording and an alerting mechanism. The Raspberry Pi 3 Model B+implemented in the design is the latest model of the Raspberry pi and the most advanced. The Raspberry pi 3 Model B+ Specification are Broadcom BCM2837B0, Cortex-A53 (ARMv8) 64-bit SoC at 1.4GHz, 1GB LPDDR2, SDRAM $2.4 \mathrm{GHz}$ and $5 \mathrm{GHz}$ IEEE $802.11 \mathrm{~b} / \mathrm{g} / \mathrm{h} / \mathrm{ac}$ wireless LAN, Bluetooth 4.2, Bluetooth Low Energy (BLE) Gigabit Ethernet over USB 2.0 (maximum throughput $300 \mathrm{Mbps}$ ) Extended 40-pin General-Purpose Input/Output (GPIO) header Full-size HDMI, 4 USB 2.0 ports, Camera Serial Interface (CSI) port for connecting a Raspberry Pi camera, Display Serial Interface (DSI) port for connecting a Raspberry Pi touchscreen display, 4-pole stereo output and composite video port, Micro SD port for loading the operating system and storing data, 5V/2.5A DC power input, Power-over-Ethernet (PoE) support. The Raspberry pi 3 Model B+ is shown in Figure 1.

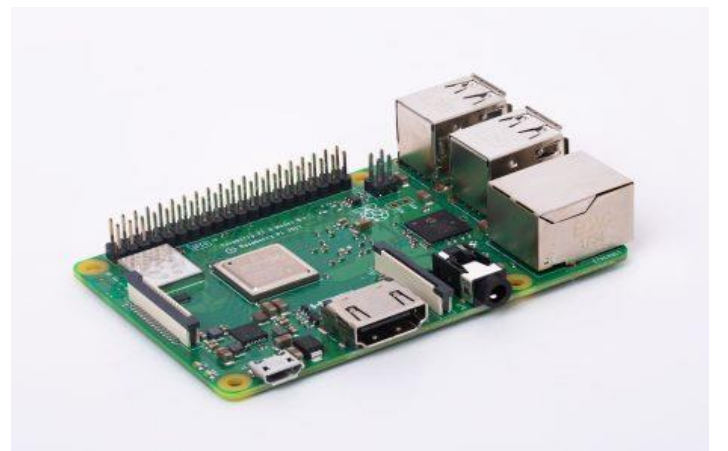

Figure 1: Picture of the Raspberry Pi 3 Model B+ [6]

\section{(b) Passive Infrared Motion Sensor}

All objects, living or not, whose temperature is anything above absolute zero emits infrared radiation. This radiation (energy) is invisible to the human eye but can be detected by electronic devices designed for such a purpose. The word "passive" in the term "passive infrared" refers to the behaviour of the detector, which receives infrared radiation passively [7]. The sensors could gather data relative to the acceleration, velocity, and position of the object. A PIR motion detector consists of a passive IR receiver that measures ambient temperature. If this temperature changes rapidly, like when a person walks across its field of view, an alarm condition is sent to the controller. This functionality is quite distinct from active infrared detector in the sense that the passive infrared detector doesn't emit the infrared ray; it receives the infrared ray that is emitted by objects. PIR Motion detector is very easily interfered by the variation of heat sources and sunlight, on that note, PIR Motion detector is considered to be more suitable for the indoor movement detection, which is characterized of closed environment [7]. Figure 2 illustrates a typical schematic diagram of the passive infrared motion sensor.

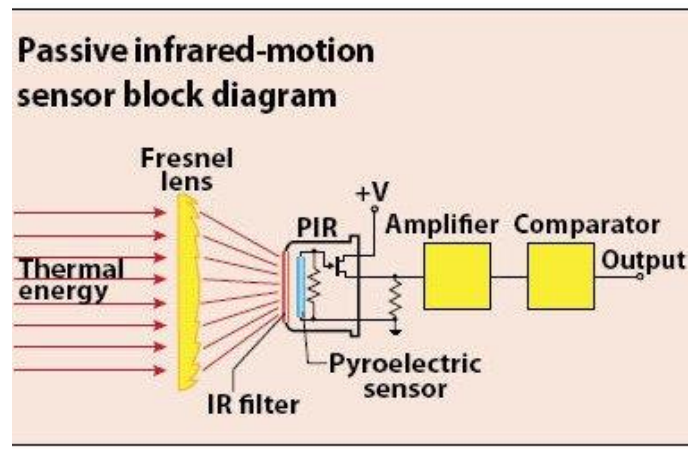

Figure 2: A Passive Infrared Motion Sensor [7]

\section{(c) Pi camera module}

The Pi camera is a device which can capture image and video. Currently, it is the only camera made specifically for the Raspberry Pi device [8]. To initialize and activate the camera settings, specific configurations are required, including a Python script written to enable the device capture pictures. 


\section{(d) Micro SD card}

A $32 \mathrm{~GB}$ card was used for the installation of the operating system, booting, and in storage/memory for the recorded videos. Raspbian software was installed in the system to interface pi camera, buzzer and PIR sensor through Raspberry pi device.

\section{(e) Buzzer $5 \mathrm{~V}$}

A buzzer or beeper could be described as an audio signaling device which may be mechanical, electromechanical, or piezoelectric. The buzzer used in the design security system is the Buzzer $5 \mathrm{~V}$. It operates as follows: By applying $3 \mathrm{~V}$ to $5 \mathrm{~V}$ to the buzzer module, a loud 2 $\mathrm{KHz}$ BEEP sound would be heard. Unlike a plain piezoelectric buzzer, this buzzer does not need an AC signal. Inside the Buzzer $5 \mathrm{~V}$ is a piezoelectric material and the driver circuitry that makes it oscillate at $2 \mathrm{KHz}$.

\section{(f) Web Server}

A web server is a system that delivers content or services to end users over the internet. A web server consists of a physical server, server operating system (OS) and software used to facilitate HTTP communication [9]. The web server used in this project is the XAMPP software. This software allows a personal computer to be used as a local server or local host. An online web server can also be used. A Database was created for the user and the video contents to be stored in the server. The web server is composed of the following main pages: (a) Index page: usually used to refer to the front page, web server directory index or the main web page of a website. (b) Register Page: describes location where first time user can fill in their details for onward storage in the database. Login page: indicates where a registered user fills details which is subsequently verified before granting the user login access and Dashboard page: demonstrates where the information (video) sent is displayed alongside with the uploaded time frame.

\section{METHODOLOGY}

The sensor implemented in the design is the Passive Infrared (PIR) motion sensor HC-SR501. It uses infrared sound and vibration to detect moving objects or people. When any motion is detected, the PIR sensor outputs a HIGH signal on its output pin, which can be read by a microcontroller and then drive a transistor to switch a higher current load. The detection range is 7 meters by 140 (degrees) coning angles. It has a delay time of 16 seconds but adjustable. The ambient temperature is $253 \mathrm{~K}-323 \mathrm{~K}$. It was powered directly from the $\mathrm{Pi}$ through the $5 \mathrm{~V}$ dc supply pin. Its output was connected as the input to the programmable GPIO pin.

Raspberry Pi camera is attached to a small printed circuit board which is then connected to the Raspberry pi via a camera board. The camera can deliver a clear image, $5 \mathrm{mp}$ resolution and video recording at $1080 \mathrm{HD}$. It is connected to the port opposite of Ethernet port in the Raspberry pi. The ribbon cable which is extendable enables the attachment of Printed Circuit Board (PCB) and allows for the connection of the camera to the pi via its port. The device is enabled by importing and installing the camera from the Raspbian operating system. The Raspbian software is installed in the system to interface pi camera, buzzer and PIR sensor through the Raspberry pi.

Figure 3 demonstrates the configuration of the Raspberry pi and the connections to the PIR sensor, Buzzer and LED. It also shows the interface of the raspberry pi to the web server and the interactions with the database.

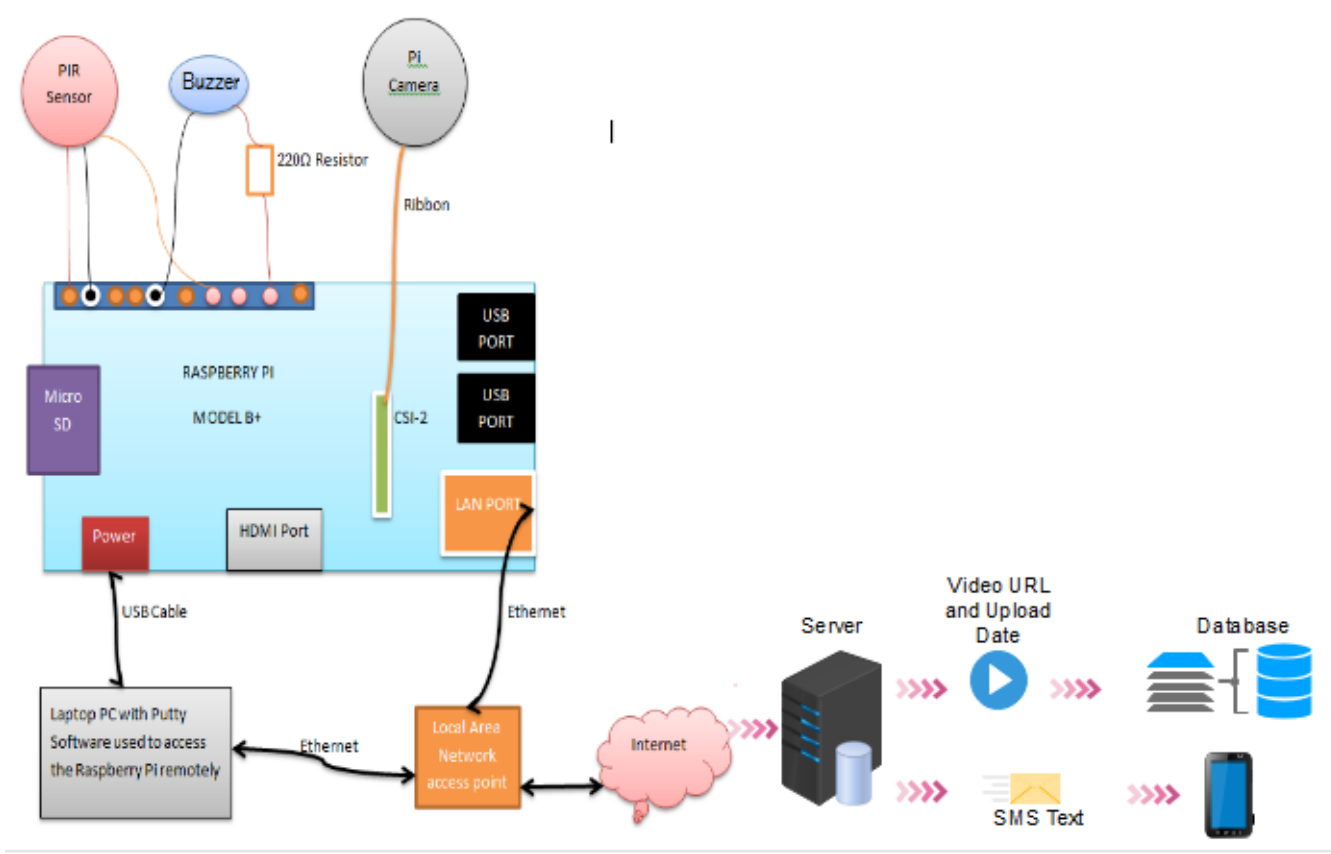

Figure 3: System Architecture of the Proposed Security System 
A Light Emitting Diode (LED) was used to indicate an intrusion. This was designed to be controlled through the action of a PIR sensor. This device was connected to the GPIO pin through a $220 \Omega$ resistor. The buzzer was incorporated in the design to be controlled by the action of a PIR sensor. It is connected together with the LED to the GPIO pin through a $220 \Omega$ resistor. When the LED is switched ON, the buzzer is also activated. Thus, it resonates, indicating that an intrusion has occurred. The hardware connection between the Raspberry pi, PIR sensor, buzzer, LEDS and resistors was configured on the breadboard using jumper wires as shown in figure 10 . The Raspberry pi was programmed to actuate other connected devices when it receives a "logical 1" input from the PIR sensor. It infinitely loops the process until it is powered off. A flowchart representing the PIR input and output drill is shown in Figure 4.

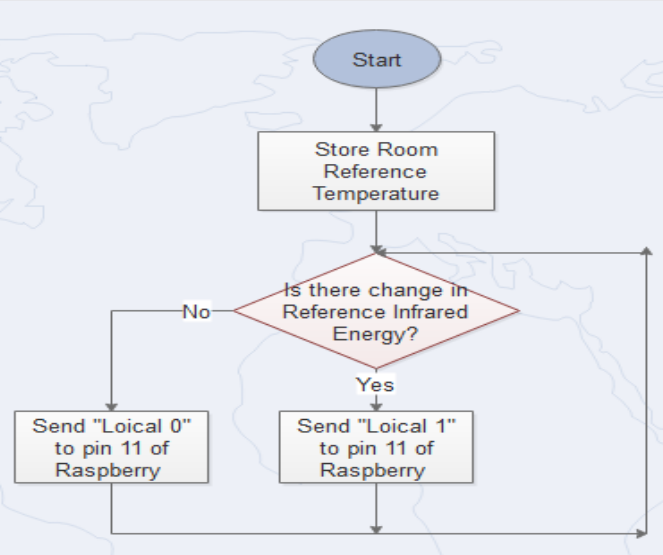

Figure 4: The flow mechanism of the PIR sensor Input and Output
On reception of logical 1 input, the raspberry actuates the buzzer, LED and camera. The flow chart is represented in Figure 5.

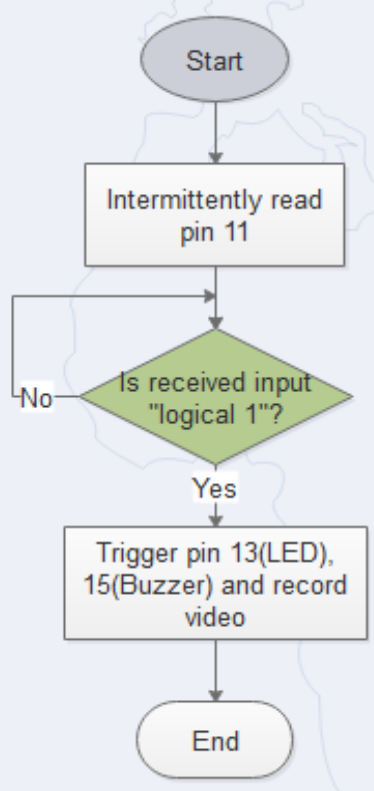

Figure 5: Flowchart showing the Raspberry Actuation of Peripherals Process

After few seconds when the video had recorded the events, the next stage was the conversion of the processed video information from the default h264 format to MP4 format before being uploaded to the server with the video list automatically displayed on the website.

Figure 6 illustrates the sequence of events from intrusion point up to the receiving point. The system algorithm was realized using a Python script.

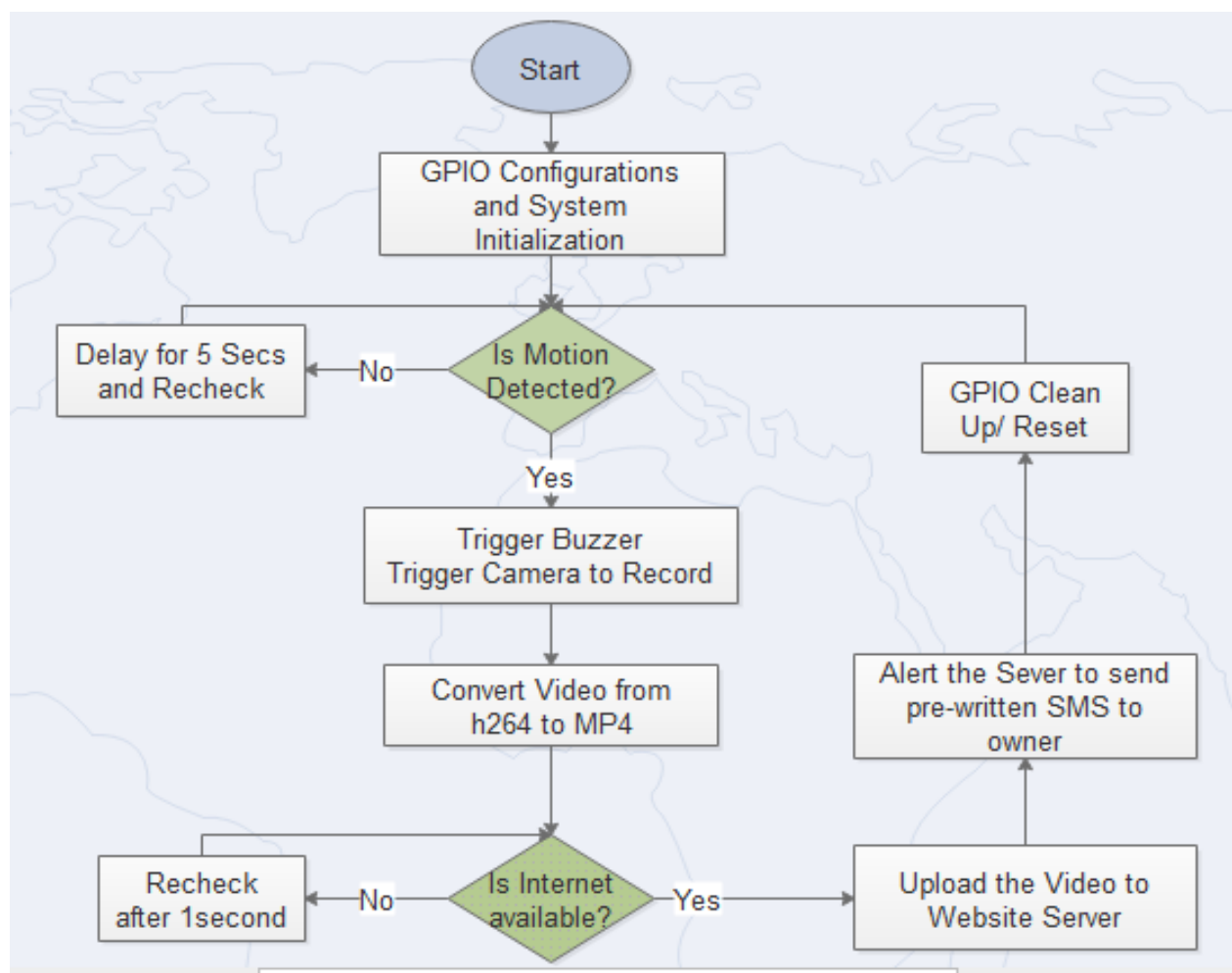

Figure 6: Flow chart representation of the proposed security system 


\section{A. User Authentication and Access Granting Process}

To access the list of uploaded videos, the user has to login using already registered username and password, the database compares the input and stored details before granting or denying access. Figure 7 shows the flow chart of the authentication process.

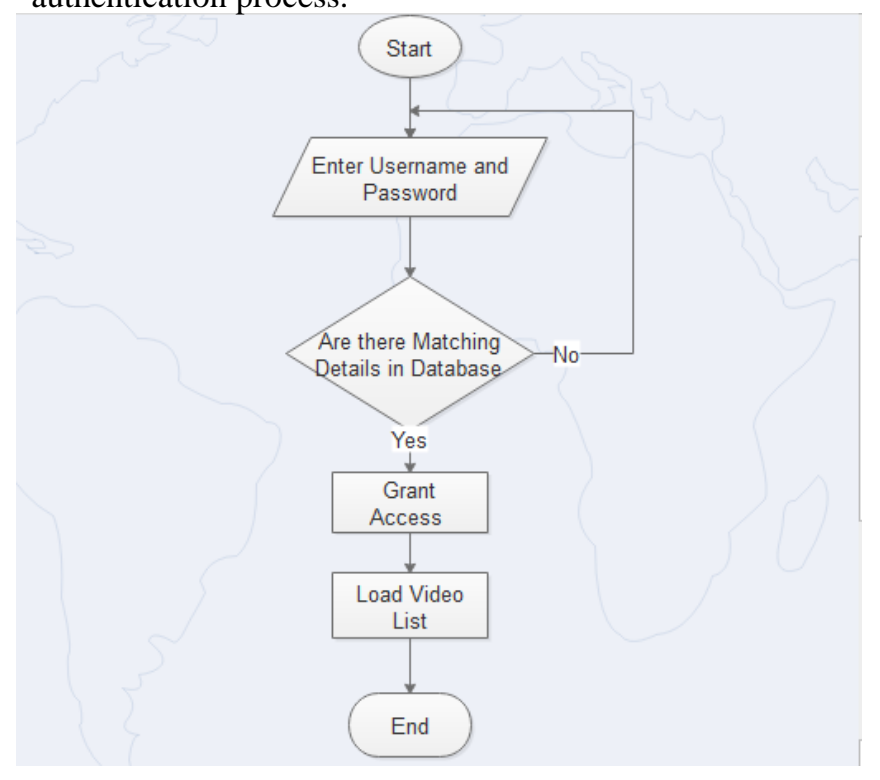

Figure 7: User Authentication and Access Grant Flow Chart

\section{B. System Initialization and Configuration}

The Raspberry pi configuration starts with the installation of the Raspbian Operating System (ROS) in the 32GB SD
Card. The SD card was then slotted into the Raspberry pi. A router was used in the initialization and configuration process. The router was connected to the Raspberry pi via an Ethernet cable. The Raspberry pi connects to the router's hotspot along with a computer. A Local Area Network (LAN) was formed by these three devices. The computer pings the Raspberry pi by port scanning, and connects to its Internet Protocol (IP) address using the Secure Socket Shell (SSH) protocol.

The command "sudo raspi-config" was typed in the Command Line Interface (CLI) of the raspberry pi. This opens up a list of options and the network settings was selected. In the network settings, the configuration of the Raspberry pi was enabled to create an interface to only the particular hotspot with username and password. This settings was saved followed by the shutdown of the Raspberry pi. The router was switched off and disconnected from the Raspberry pi. Whenever the raspberry pi is powered $\mathrm{ON}$, it automatically connects to the specified hotspot and devices such as phones and computers which are interfaced to it. After the configuration, the Raspberry pi can then through the network perform the tasks of importing Python libraries and packages, Pi camera setting/configuration and the GPIO settings and its pin initialization.

\section{Design Specifications}

The components used in the proposed security system has some characteristic specifications for operating voltage, current, temperature and even dimensions etc. These specifications are shown in Table 1.

Table 1: Design Specifications

\begin{tabular}{|c|c|c|c|c|c|}
\hline Components & $\begin{array}{l}\text { Operating } \\
\text { voltage }(V)\end{array}$ & $\begin{array}{l}\text { Operating } \\
\text { current }\end{array}$ & $\begin{array}{c}\text { Operating } \\
\text { temperature }\left({ }^{0} \mathrm{C}\right)\end{array}$ & Dimensions & Resolution \\
\hline Raspberry pi & $3.3 \mathrm{~V}-5 \mathrm{~V}$ & $700 \mathrm{~mA}-2000 \mathrm{~mA}$ & $0^{\circ} \mathrm{C}-85^{\circ} \mathrm{C}$ & N/A & N/A \\
\hline PIR sensor & $5 \mathrm{~V}-20 \mathrm{~V}$ & $50 \mathrm{uA}$ & $-15-70^{\circ} \mathrm{C}$ & $32 \mathrm{~mm} \times 24 \mathrm{~mm}$ & N/A \\
\hline Buzzer & $3-24 \mathrm{~V}$ & $50 \mathrm{~mA}$ & $-20-+70^{\circ} \mathrm{C}$ & N/A & N/A \\
\hline LED & $1.2-3.6 \mathrm{~V}$ & $10-30 \mathrm{~mA}$ & $55-85^{\circ} \mathrm{C}$ & N/A & N/A \\
\hline Adaptive plastic cover & N/A & N/A & N/A & 6" x 9" & N/A \\
\hline Raspberry Pi Camera & $3.3 \mathrm{~V}-5 \mathrm{~V}$ & $70 \mathrm{Ma}$ & $60^{\circ} \mathrm{C}$ & $25 \mathrm{~mm} \times 25 \mathrm{~mm} \times 9 \mathrm{~mm}$ & $\begin{array}{l}\text { Still-5 Mega Pixel } \\
\text { Sensor - } 2592 \text { x } 1944 \text { pixels }\end{array}$ \\
\hline
\end{tabular}

\section{Database and Web Server Protocol}

A database is a collection of information organized in such a way that a computer program can quickly select desired pieces of data [10]. The authors of [11] further defined it as any collection of data or information that is specially organized for rapid search and retrieval by a computer. A Database Management System (DBMS) extracts information from the database in response to queries. The database created for the security system consists of two stands namely; user and pivot. The user contains information such as username, password, E-mail, phone number, Address. This information is from the registered user to enable the personnel have access to the video information. The pivot contains the URL (video link) and the upload date. This helps to distinguish the videos sent by the raspberry pi to the server. After the creation of the database, the software "brackets" was used to create a PHP

script called "register". This enables the user of security system to register with the system and have the details saved in the database. Whenever the user logs in, proper authentication is carried out to prevent unauthorized users from having access to the system. Also two other PHP scripts work in conjunction with the register PHP script. The first PHP script is the connect PHP script which ensures that connection between the server and database is made. The second is the "adduser" PHP script which ensures that users who have filled in their details are added to the database.

A login PHP script is written to allow registered users to have access to video feeds of the security system. A dashboard PHP script is written to display the video link (URL) along with the date, the associated video is uploaded to the webserver by the raspberry pi and by clicking on the video link, a video page is opened, the video of the intrusion is displayed. An upload PHP script is also written to receive the video feeds sent by the Raspberry pi, the time it was sent and also to store the URL and the timed information in the database including the video feeds.

To enable communication between the raspberry pi and the web server, a python library called "requests" is imported in Raspberry pi python script to make hypertext transfer 
protocol (HTTP) connection to the web server. A python code is written to send the video feeds of the intrusion from the raspberry pi to the webserver and on the webserver end; there is a PHP script to accept the video feeds and to store it in a file system, and save the video details in the database. The web server is also programmed to send a Short Message Service indicating that an intrusion has occurred to the user's phone number stored in the database.

\section{E. Human Detection}

The Human detection fragment of the designed security system is entirely dependent on the python program script, responsible for detection of motion and actuation of the connected devices. The PIR sensor responsible for the detection of motion adjusts itself to the infrared signature of its surroundings and keeps watching for any changes. In the absence of motion, the LED indicator will remain off and the buzzer does not sound, and the program will continue updating the surroundings. If the sensor detects movement, the frame for motion detected will be the input frame to the process of human detection, and consequently, the motion detection indicator will light up and the buzzer sounds.

\section{RESULTS}

Tables 2, 3 and 4 show the sensitivity of the PIR sensor at maximum, intermediate and minimum respectively.

Table 2: Results for maximum sensitivity of the PIR sensor

\begin{tabular}{|l|l|l|l|}
\hline $\begin{array}{l}\text { Distance in } \\
\text { meters }\end{array}$ & $\begin{array}{l}\text { LED } \\
\text { ON/OFF }\end{array}$ & Alert send & Ref. \\
\hline 15 & OFF & NO & None \\
\hline 10 & OFF & NO & None \\
\hline 8 & ON & YES & None \\
\hline 6 & ON & YES & None \\
\hline 5 & ON & YES & None \\
\hline 4 & ON & YES & None \\
\hline 3 & ON & YES & None \\
\hline
\end{tabular}

Table 3: Results for medium sensitivity of the PIR sensor

\begin{tabular}{|l|l|l|l|}
\hline $\begin{array}{l}\text { Distance in } \\
\text { meters }\end{array}$ & $\begin{array}{l}\text { LED } \\
\text { ON/OFF }\end{array}$ & Alert send & Ref. \\
\hline 15 & OFF & NO & None \\
\hline 10 & OFF & NO & None \\
\hline 8 & OFF & NO & None \\
\hline 6 & ON & YES & None \\
\hline 5 & ON & YES & None \\
\hline 4 & ON & YES & None \\
\hline 3 & ON & YES & None \\
\hline
\end{tabular}

Table 4 : Results for low sensitivity of the PIR sensor

\begin{tabular}{|l|l|l|l|}
\hline $\begin{array}{l}\text { Distance in } \\
\text { meters }\end{array}$ & $\begin{array}{l}\text { LED } \\
\text { ON/OFF }\end{array}$ & Alert send & Ref. \\
\hline 15 & OFF & NO & None \\
\hline 10 & OFF & NO & None \\
\hline 8 & OFF & NO & None \\
\hline 6 & OFF & NO & None \\
\hline 5 & OFF & NO & None \\
\hline 4 & ON & YES & None \\
\hline 3 & ON & YES & None \\
\hline
\end{tabular}

When intrusion of a human is confirmed in the field of view of the sensor, the Passive Infrared sensor triggers the pi camera through the Raspberry pi. The raspberry pi directs instructions to the pi camera to carry out video recording and consequently send it to the web server. Figure 8 shows a captured intrusion video.

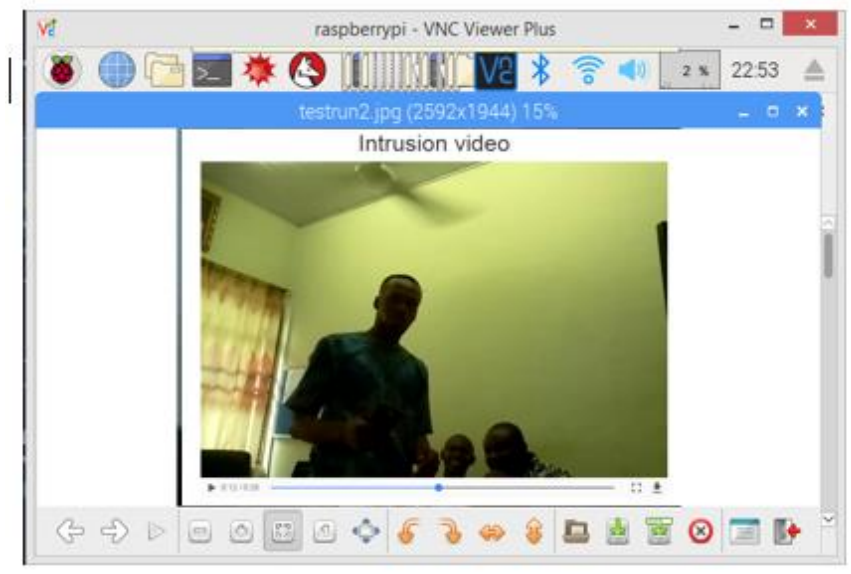

Figure 8: Viewed intrusion Video

After an intrusion is detected by PIR sensor, the Raspberry pi instructs the pi camera to record a video, and the buzzer to sound. The Raspberry pi also sends a Short Message Service (SMS) to the specified user of the system alerting the personnel of an intrusion occurrence and also referring the user to the web application to view and confirm the identity of the intruder for further actions. Figure 9 shows experimental tests of received SMS on intruders while Figure 10 demonstrates the configured designed prototype.

$1 \stackrel{H}{H} \square .114 \%$ 8:32 am

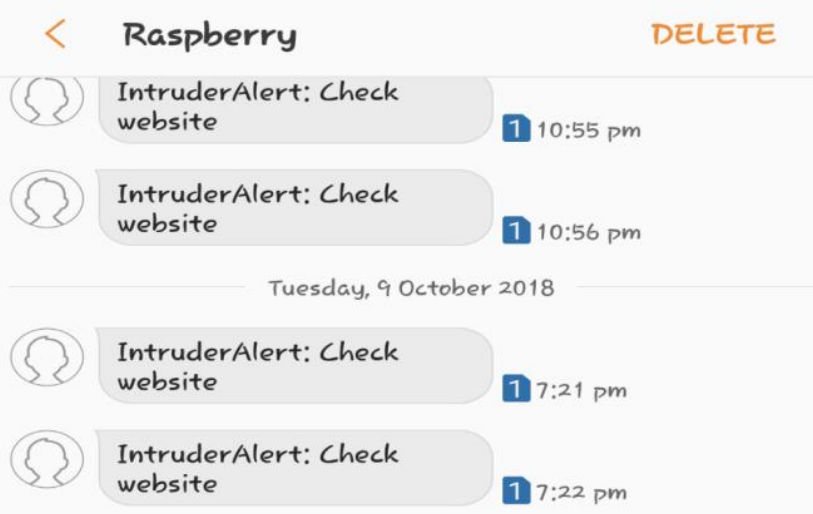

Fig 9: Experimental tests of Received SMS on intruders

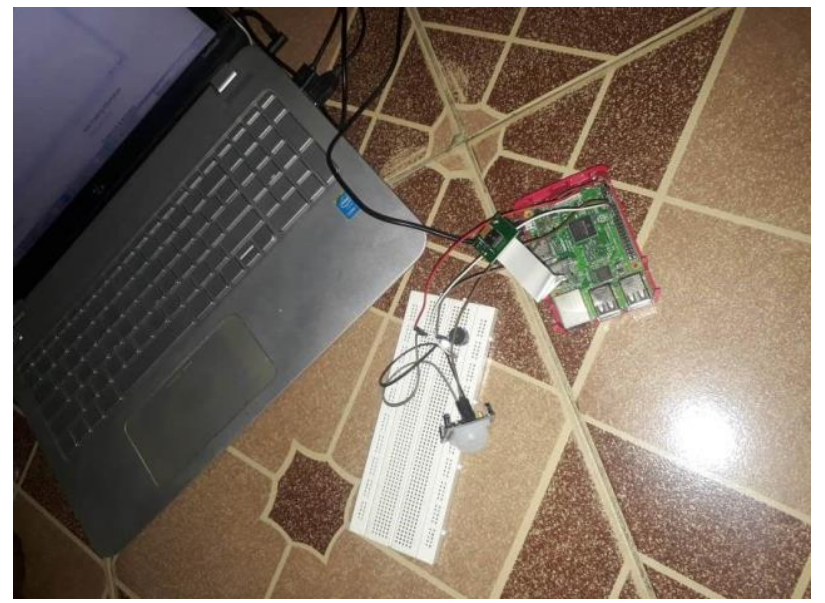

Figure 10: the designed structured prototype 


\section{DISCUSSION}

The hardware and the software parts of the proposed security system are interfaced to achieve the overall objective of home security system. While the hardware part contains devices that make the surveillance possible and achievable, the software drives the operations and enables the functioning of the interconnected devices. The primary objective of the project is to ensure the security of the home while at the same time managing the costs associated with the installation of the surveillance system.

The sensitivity of the PIR sensor is at a distance of between 6 to 10 meters. A meticulous positioning of the sensor enhances its operations. For example, the placement of the sensor in such a way that an intruder walks across its field of vision improves its performance in motion detection as compared to the positioning when an intruder walks straight towards the sensor. The pi camera captures any movements within this range of the sensor coverage.

The Raspberry pi controls the programs necessary for video recording and transmitting to the web server. When the PIR sensor detects motion, raspberry pi enables the Pi camera to capture and store the image.

The system design alerts the homeowner about any intrusion in the home via an SMS which quickly refers him/her to the web application to watch the recorded video. At this instant, the owner of the home can take necessary steps to avoid any further damage to the property.

\section{CONCLUSION}

With an ever improving awareness of the importance of security in both residential and commercial buildings, homeowners, employers and workers are on the lookout for an efficient surveillance system which is cost-friendly. Detection of intrusion into the home or office is made possible using passive infrared sensors. The sensor uses the infrared radiations which alters as a result of human motion across its field of movement. When movement is detected, the raspberry pi enables the buzzer to alert the personnel on a possible intrusion. The recorded video is then sent to a specified online web server, the homeowner can then login to watch the recorded videos. The web server is also tasked with sending a Short Message Service (SMS) to owner's mobile phone. The system designed is energy efficient and is applicable particularly in areas with low energy supply as a result of inadequate electricity supply. It enables the owner to be aware of the security situation at home or office. The proposed security system is recommended for residential applications due to its efficiency and effectiveness in home surveillance application.

\section{REFERENCES}

[1] VinTech Systems, "Back to Basics: Where Did the Burglar Alarm Come From?".

Available:https://vintechnology.com/2011/04/08/back-to-basics-where -did-the-burglar-alarm-come-from/ 2011 [Accessed: 18 June 2018.]

[2] Administrator, "GSM Based Home Security Alarm System Using Arduino" 2016. Available:

https://www.electronicshub.org/arduino-gsm-home-security-alarm-system/ 2016. [Accessed: 4 July 2018]

[3] Bhavani A., Jami T. and Ashok G., "Low Cost Smart Security Camera with Night Vision Capability Using Raspberry Pi and PIR Sensor". International Journal of Advanced Technology and Innovative Research(IJATIR)Vol. No. 8, No. 21, pp. 4053-4056, 2016.
[4] Iyapo K.O., Odo E.A, Fasunla O.M, Egbuwalo S.A and Raimi O.A, "Design and Construction of an Automatic Home Security System based on GSM Technology and Embedded Microcontroller Unit", American Journal of Electrical and Computer Engineering. Vol 1, No. 1,pp. 25-32, 2017

[5]Khairul M.D., "Home Alarm Sytem Using Detector System", Department of Electronic Engineering:Universiti Teknikal Malaysia Melaka, 2009.

[6]Available:https://www.raspberrypi.org/products/raspberry-pi-3-model-b -plus [Accessed: 02 August 2018]

[7] RobotsCraft technology, "Introduction to PIR Sensor and Integrating It With Arduino,".

Available:http://www.instructables.com/id/Introduction-to-PIR-Sensor -and-Integrating-It-With/2016. [Accessed 03 August 2018].

[8] Sheshai S., "Raspberry Pi Based Security System," Nairobi, 2016.

[9] Available: https://www.techopedia.com/definition/4928/web-server

[10] Beal V. "database". Available: https://www.webopedia.com/TERM/D/database.html [Accessed: 21 September 2018].

[11] Britannica "Database". Available: https://www.britannica.com/print/article/152195[Accessed:21 September 2018. 